\title{
Food matters: how the microbiome and gut-brain interaction might impact the development and course of anorexia nervosa
}

\author{
Beate Herpertz-Dahlmann ${ }^{1} \cdot$ Jochen Seitz $^{1} \cdot$ John Baines $^{2}$
}

Received: 25 July 2016 / Accepted: 10 January 2017 / Published online: 31 January 2017

(c) The Author(s) 2017. This article is published with open access at Springerlink.com

\begin{abstract}
Anorexia nervosa (AN) is one of the most common chronic illnesses in female adolescents and exhibits the highest mortality risk of all psychiatric disorders. Evidence for the effectiveness of psychotherapeutic or psychopharmacological interventions is weak. Mounting data indicate that the gut microbiome interacts with the central nervous system and the immune system by neuroendocrine, neurotransmitter, neurotrophic and neuroinflammatory afferent and efferent pathways. There is growing evidence that the gut microbiota influences weight regulation and psychopathology, such as anxiety and depression. This article reviews how the gut-brain interaction may impact the development and course of AN. A "leaky gut", characterized by antigens traversing the intestinal wall, was demonstrated in an animal model of AN, and could underlie the low-grade inflammation and increased risk of autoimmune diseases found in AN. Moreover, starvation has a substantial impact on the gut microbiome, and diets used for re-nutrition based on animal products may support the growth of bacteria capable of triggering inflammation. As there is currently no empirically derived agreement on therapeutic re-nourishment in AN, this review discusses how
\end{abstract}

This article is part of the Focused Issue "The role of nutrition in child and adolescent onset mental disorders" of European Child and Adolescent Psychiatry.

Beate Herpertz-Dahlmann

bherpertz@ukaachen.de

1 Department of Child and Adolescent Psychiatry, Psychosomatics and Psychotherapy, University Clinics, Technical University RWTH, D-52074 Aachen, Germany

2 Institute for Experimental Medicine, Christian-AlbrechtsUniversity of Kiel and Max Planck Institute for Evolutionary Biology, Plön, Germany consideration of gut-brain interactions may be important for treatment regarding the determination of target weight, rapidity of weight gain, refeeding methods and composition of the diet which might all be of importance to improve long-term outcome of one of the most chronic psychiatric disorders of adolescence.

Keywords Anorexia nervosa $\cdot$ Microbiome $\cdot$ Gut-brain interaction $\cdot$ Starvation $\cdot$ Autoimmune disease

\section{Introduction}

The Hippocratic concept of positive health dates back to the fifth century B.C. and postulates that adequate nutrition is an important component of a long and healthy life [1]. Nutrition comprises macronutrients (carbohydrates, proteins and fat) and micronutrients (vitamins and minerals) to supply energy and enable an organism to grow and function properly. Although most people consider sufficient and balanced eating as a basic drive to maintain well-being, anorexia nervosa (AN) is characterized by insufficient food intake and poor diet, which lead to a significantly low body weight and severe danger to the individual's health. Additionally, patients with AN suffer from severe weight phobia; in many but not all cases, there is a lack of recognition of the seriousness of the illness [2].

Some 30 years ago, the aetiology of AN was explained by a psychosomatic family model [3, 4]. Herein, nutritional rehabilitation and weight gain were not considered to be of primary importance to the healing process. Today, it is well-known that a higher body mass index (BMI) at the end of treatment correlates with a better overall outcome [5] and that a normalization of body weight is necessary to prevent severe somatic sequelae such as osteoporosis 
and infertility $[6,7]$. Moreover, it is well-established that weight gain is a potent agent against comorbid mental disorders of AN, especially depression $[8,9]$.

Most mental disorders emerge by an interaction between a person's biological disposition and environmental influences, i.e. by an interaction of "nature" and "nurture". In $\mathrm{AN}$, the latter is to be taken literally. In animals and healthy individuals, eating is both a motivating and appetitive behaviour that is highly reinforced and rewarded by certain neurocircuits in the brain (for a review, see [10]). However, this "core eating network" [10] seems to be severely affected in patients with eating disorders, especially AN. These individuals seem to process food cues differently in comparison to healthy eaters, exhibit decreased anticipation of food taste and often show strong regulatory responses enabling control and restraint of food intake [10]. However, it remains unclear whether these changes are primary in origin, and thus a cause of the eating disorder, or whether they appear during the illness as secondary effects.

There is emerging evidence of important links between the gut microbiome and the CNS, which might be depicted as "a gut feeling for the brain" [11] (for a review, see [12]). Humans sustain a symbiotic relationship with the microbiome in their gut, which consists of approximately $10^{14}$ cells; thus there are ten times more bacteria than cells in the average human body [13]. The highest bacterial concentrations are found in the colon with approximately $10^{12}$ per gram [14]. We supply our microbiota with food, and they compensate us with important health benefits in relation to digestion, growth and defence against pathogens [11, 15].

We have recently learned that malnutrition and longterm dieting have a substantial and reproducible effect on the gut microbiome and its impact on the brain, which is likely related to the development of psychopathology and psychiatric disorders [15]. There is growing evidence that the gut microbiome also plays an important role in the development and persistence of eating disorders, especially AN [16].

Among all age groups, less than $50 \%$ of patients with AN fully recover [17], and the mortality risk is the highest of all psychiatric disorders [18]. Medication has a very limited, if any role in the treatment of AN, and psychotherapeutic interventions are only moderately effective (for a review, see [19]). To make progress in treatment, a more "brain-directed therapy" [20] is likely necessary to overcome this often disabling disease.

Thus, the aim of this article is to present recent findings on the gut-brain interaction that may be of relevance to the pathophysiology of eating disorders, and for AN in particular. In part because this research is still at an early stage, several results are contradictory, and most findings are based on animal models that may not be easily transferred to humans. While we do not fully review all substantial aspects of gut-brain interaction for weight regulation or psychiatric disorders, our assessment aims to focus on the influences of malnutrition, the process of refeeding and their presumable impact on the course of AN. "Nutritional medicine" and microbiota-modulating strategies may be promising determinants of the healing process and outcome of AN.

\section{Communication pathways between the gut microbiome and the brain}

The gut is the most heavily bacterially colonized area of the human body, with greater bacterial numbers in the colon than in the upper intestine (see above), in addition to differences in composition between the bacteria in the gut lumen and those near the mucus layer [21]. One of the most important "tasks" of the gut microbiome is contributing to the protection of the intestinal barrier, which must prevent the passage of pathogenic microorganisms and toxic substances into systemic circulation. Certain periods of life seem to involve particular vulnerability to changes in gut permeability, e.g. early life and old age [22]. There are several pathways by which changes in the intestinal barrier might impact brain homeostasis, particularly neuroendocrinological alterations, neurotransmission, neurogenesis and neuroinflammation, either by direct passage to the brain or by the vagus nerve, which acts as the main pathway from the lumen of the gut to the nucleus solitarius in the medulla oblongata [11].

\section{Neuroendocrinological pathways-Hypothalamic- Pituitary-Adrenal (HPA)-axis}

In animal models and human studies, the experience of stress is linked to an increase in the permeability of the intestinal barrier, which might be mediated by-among other factors-hypothalamic hormones, especially CRH [22]. Acute stress increases gastrointestinal and bloodbrain barrier permeability through the activation of mast cells, which express high-affinity receptors for CRH [15]. Conversely, in germ-free (GF) rodents, higher levels of ACTH and CRH are observed after exposure to stress compared to conventionally colonized animals, suggesting that the gut microbiome contributes to the downregulation of the HPA-axis. Notably, this effect appears dependent on specific bacterial taxa, as only certain species such as Lactobacillus salivarius were able to attenuate the stress response [11].

In humans, even relatively small acute stress situations such as public speaking are followed by an increase in intestinal permeability, although only in those who also respond with elevated cortisol levels [23]. 
AN is associated with elevated serum, urinary and salivary cortisol levels in the acute state, as well as with a lack of cortisol suppression on overnight dexamethasone and dexamethasone suppression-CRH stimulation testing [24, 25]. Thus, it may be hypothesized that AN-specific aberration of the HPA-axis could contribute to the dysfunction of the intestinal barrier that is observed in an animal model of AN (see below, [26]).

\section{Neurotransmission}

It is increasingly recognized that gut microorganisms have a notable effect on the development and regulation of peripheral and central serotonergic function, especially in the hippocampus. Yano et al. [27] propose a model in which products of microbial fermentation, e.g. shortchain fatty acids (SCFA, see below) and bile acids (see below), directly act on enterochromaffin cells, and thus promote the release of serotonin, which influences gastrointestinal mobility and platelet function. GF mice display depressed levels of serotonin in plasma compared to conventionally raised mice $[28,29]$. However, they display less anxiety- and depression-like behaviour than naturally raised mice [30, 31]. Anxiety and depression are closely linked to disturbed serotonin metabolism [32]. In contrast to the findings by Wikoff et al. [28] in plasma extracts of GF mice, significantly increased hippocampal levels of 5-hydroxytryptamine (5-HT) and 5-hydroxyindoleacetic acid (5-HIAA), the main metabolites of serotonin, were found in developing GF mice compared to naturally raised mice. A sustained absence of microorganisms in these animals was observed to induce a 1.3 -fold increase in 5-HT, which compares to that induced by antidepressants such as SSRIs (for a review, see Clarke [33]). This increase appears to be sex-specific as it was only found in developing male GF mice. The reasons for this sex difference are not well explored, but are probably linked to the menstrual cycle and the influence of oestrogen on the serotonergic system. Notably, elevated levels of 5-HT are maintained when these mice are colonized with a normal microbiota later in life, which also demonstrates that the early life period plays an important role in configuring the gut-brain interaction [33].

A recent study in patients with major depression demonstrated that patients differed from normal controls in either a predominance of some potentially harmful gut bacteria or a reduction of beneficial bacterial groups [34]. Further, a large evaluation of medical record-based data in the UK showed that repeated use of antibiotics was associated with a significant increase in depressive and anxious symptoms [35].

It is suspected that the serotonergic system is severely altered in AN. During the active state of the illness, patients have a significant reduction in CSF-5-HIAA in comparison to healthy controls; in long-term weight-restored patients, CSF-levels of 5-HIAA are elevated, which is generally related to disorders of anxiety and obsession [36]. A dysfunction of the serotonergic system involving 5-HT-receptors and 5-HT-transporters in AN was also confirmed by brain-imaging studies (for a review, see [37]). However, medications targeting serotonergic dysfunction, such as SSRIs, are not proven effective in AN (for a review, see [38]).

\section{Neurogenesis}

Brain-derived neurotrophic factor (BDNF) is a nerve growth factor known to influence neuronal development, increase synapse plasticity especially in the hippocampus, and confer protection against stress-induced damage [39]. BDNF function is linked to mood, stress tolerance and cognitive function such as memory. Recent animal research suggests that the gut microbiome may influence the expression of BDNF in the brain, although the mechanism is not yet clear [11]. Prolonged administration of antibiotics results in a reduction [40], while pre- or probiotics (see below) seem to enhance hippocampal BDNF levels [41, 42]. In the absence of gut bacteria, the expression of BDNF in male mice is lowered in comparison to conventionally colonized mice, while it is increased by prebiotic feeding [43].

We are unaware of any studies on the association between the microbiome and BDNF in humans.

In AN, BDNF levels are reduced in acutely ill patients [44], but increase with short-term weight gain [45]. Thus, it could be hypothesized that diet-associated changes in the microbiome contribute to different stage-related levels of BDNF in the brains of AN patients, which might also influence cognitive functioning (e.g. psychomotor speed) [45]. Möhle et al. [46] directly showed that neurogenesis in the hippocampus was reduced in mice after antibiotic treatment, which resulted in impaired memory functions, whereas probiotic intake completely restored neurogenesis and memory functions. However, it remains to be demonstrated whether similar phenomena occur in humans.

\section{Neuroinflammation}

The intestinal barrier normally prevents pathogenic microorganisms from spreading into systemic circulation. However, the so-called "leaky gut" (increased intestinal permeability) might facilitate the transfer of potentially pathogenic members of the microbiota (i.e. "pathobionts"), metabolites, toxins or lipopolysaccharides from the gut lumen to the lamina propria and on to the mesenteric lymph nodes, from which they may reach systemic circulation, especially in the case of an aberrant immune response [12, 15, 47]. 
This phenomenon was observed both in animal models for depression [48] and in depressed humans [47]. Once in circulation, the bacteria and other gut luminal contents stimulate the output of pro-inflammatory peripheral and central cytokines, the latter influencing neuronal function. Enhanced levels of IgA- and IgM antibodies against bacterial lipopolysaccharide, the structural component of the external membrane of Gram-negative bacteria, are observed in depressed patients, especially in those with chronic disorders [49]. However, it is not yet clear what comes first; increased gut permeability could induce mucosal inflammation followed by systemic inflammation; conversely, systemic inflammation could disturb intestinal barrier function, thus leading to an increased bacterial translocation and fuelling of systemic inflammation [12].

An increased risk for autoimmune disorders is observed in $\mathrm{AN}$, especially for those involving the gastrointestinal tract (see below, [50]).

\section{Short-Chain Fatty Acids (SCFAs) with neuroactive qualities}

Short-chain fatty acids mainly represent the product of fermentation of partially digestible and non-digestible carbohydrates, namely dietary fibre, by the microbiome [51]. The most important SCFAs are acetic acid, propionic acid and butyric acid, which can act as "signalling molecules" and affect the physiology of the host organism, such as influencing the ph-level of the colon, controlling the gut transit time, metabolizing glucose and modifying appetite and energy homeostasis [11, 52-54]. SCFAs are also able to cross the brain-blood barrier and impact neural circuits [55]. They are involved in regulating the expression of neuropeptides, such as PYY and ghrelin [56] and are associated with antidepressant effects in animal models via higher concentrations of BDNF.

\section{Starvation and the gut microbiome}

Mouse models provide evidence that the transfer of microbiota from genetically or nutritionally induced obese mice to GF mice can lead to obesity and associated metabolic disturbances in the host animals [57, 58]. In contrast, kwashiorkor is a severe form of acute malnutrition often observed in developping countries that is probably worsened by a protein-deficient diet. It was recently shown that the consequences of this type of malnutrition are affected by the gut microbiome and vice versa [59]. Frozen bacterial species from children with kwashiorkor could be transplanted into GF mice; the combination of the transplanted kwashiorkor species with the African diet produced significant weight loss in these mice associated with severe metabolic disturbances. Although symptoms of kwashiorkor could be alleviated by better nutrition, symptoms returned when the African diet was re-implemented.

Starvation-induced changes in the gut microbiome were also found in an acute and chronic starvation animal model using activity-based anorexia (ABA), which is the most widely utilized rodent model of AN simulating weight loss by food restriction and hyperactivity with the help of access to a running wheel (e.g. [60]). After the ABA mice lost a substantial amount of weight (approximately 20\%), a histological investigation of the colon revealed decreased thickness of the muscularis layer and significantly increased permeability of the colon [26]. These alterations observed in an animal model for AN suggest that intestinal barrier dysfunction provoked by starvation might also contribute to the pathophysiology of AN. In contrast, in an earlier study of AN patients by Monteleone et al. [61], a decrease in intestinal permeability was found. However, the authors of this earlier study used the administration of an oral sugar solution of Lactulose/Mannitol excreted in the urine for their testing, which mainly examines the permeability of the small intestine [62]. In the study of ABA mice by Jesus et al. (2014), the permeability of the colon assessed by histological analysis was increased. Moreover, the amount of the oral dose of the sugar absorption test excreted in the urine does not only depend on the permeability of the intestinal mucosa, but also on other factors, such as gastric emptying, intestinal transit time and renal clearance [62], all of which may be disturbed in AN.

In addition, several studies demonstrated an increase in intestinal permeability during exercise, which is found in the majority of patients with AN, and also represents a basic mechanism in the ABA model [63].

A disturbed gut barrier function was also found in other disorders associated with malnourishment and in volunteered fasting subjects $[64,65]$.

\section{Previous studies in AN}

Until now, our knowledge of the relevance of the gut microbiome for the symptomatology of AN is scarce. Very few studies investigated the composition of the intestinal microbiome during the acute and recovered states of AN. A single culture-based case study of an AN patient at admission identified 11 previously unknown bacterial species [66], whereas a study of 25 patients with AN found a reduced number of total bacteria and obligate anaerobes [67]. Armougom et al. [68] compared the gut microbiome in obese individuals, healthy controls (HC) and AN patients and found an increased amount of Methanobrevibacter smithii in AN [68]. 


\section{Gut-Brain Interaction}

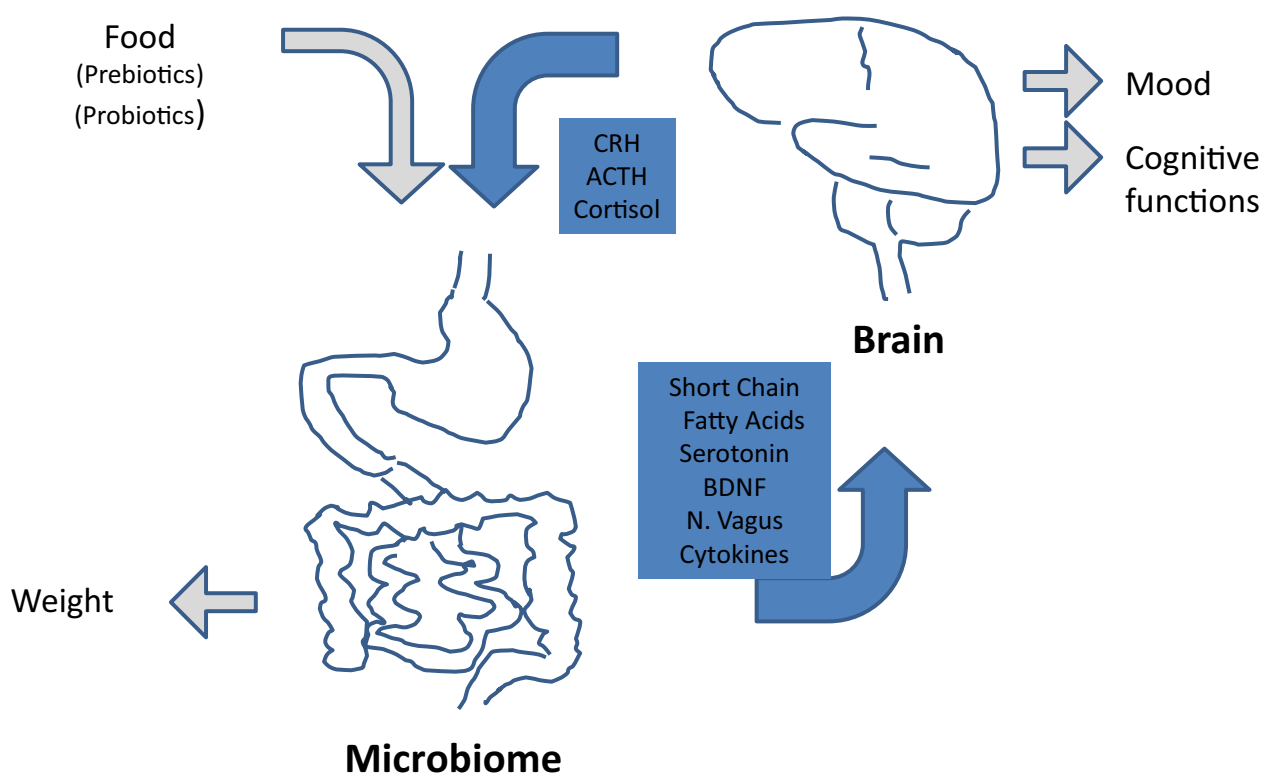

Fig. 1 Interaction between the gut microbiome and the brain. While food is one of the main influencing factors of the gut microbiome, the microbiome in turn is an important influencing factor of body weight. Multiple interactions link the microbiome with the brain: Gut originating fatty acids (SCFA's), serotonin, brain-dervied neurotrophic growth facotr (BDNF), nervus vagus stimulation and inflammatory cytokines appear to impact the brain via various pathways and

infuence mood and memory function. In turn, brain originating corticotropin-releasing hormone $(\mathrm{CRH})$, adrenocorticotropic hormone $(\mathrm{ACTH})$ and cortisol influence the gut and its microbiome. In AN, a "leaky gut" seems to increase transfer of microbiotal antigens into the systemic circulation, further increasing inflammation and influencing interactions with the brain, potentially also affecting mood and memory function

The first longitudinal results from AN were presented by Kleiman et al. [69], who analysed the faecal microbiome in a small number of female, mostly adult AN patients collected at admission $(n=16)$ and discharge after weight gain $(n=10)$ and compared it to HC samples. Weight loss at admission was of medium severity. At discharge patients (who could be reassessed twice) were still at a rather low weight. In comparison to $\mathrm{HC}$, patients with AN displayed significantly reduced alpha diversity (describing the withinsample diversity) in the state of a low BMI. After weight gain, there was an increase in alpha diversity, although it remained lower than in HC [69]. Notably, differences in overall diversity and the abundances of individual bacterial groups were significantly associated with eating disorder psychopathology and depression scores, a finding consistent with the results of other studies showing associations between the gut microbiome and behaviour (e.g. [34]).

In a very recent German study from the University of Tuebingen [70], the results of Kleiman et al. [69] were for the most part confirmed in their much larger sample of 55 individuals, which were predominantly adults with a mean BMI of approximately $15 \mathrm{~kg} / \mathrm{m}^{2}$. Although alpha diversity was lower in $\mathrm{AN}$ patients than in $\mathrm{HC}$ at admission, the difference was not statistically significant. Furthermore, as already observed in

the study by Kleiman et al. [69], there was again a significant increase in alpha diversity in AN patients after weight gain. Nevertheless, AN patients' microbiome after weight gain still resembled that of patients at the beginning of treatment more than that of $\mathrm{HC}$ in terms of beta diversity (inter-individual variability). However, BMI was still very low (mean BMI 17.7) at the second assessment point, so that the effect of a more normalized body weight could not be investigated. Importantly, the abundances of individual taxonomic groups differed significantly between AN patients and $\mathrm{HC}$, which included an increase in mucin-degraders (e.g. Verrucomicrobia and Bifidobacteria) and a decrease in butyrate-producers (e.g. Roseburia spp.) in AN patients. Mucin-degraders feed on mucus covering the intestinal wall and potentially contribute to the "leaky gut" identified in the ABA animal model of AN (39) mentioned above. Moreover, the authors analysed the production of short-chain fatty acids (see above) before and after weight gain. The concentration of the sub-group of branched-chain fatty acids, a product of protein fermentation, was increased in AN, although total SCFA production did not differ. They further note that branched-chain fatty acids can have negative effects on host physiology, e.g. by impacting PYY-releasing cells, and even psychopathology, e.g. by increasing depressive symptoms [70, 71]. 
As the peak onset of AN is in adolescence [72], the effect of young age and pubertal changes on the microbiome should be explored. An influence of age on the microbiome was just below the significance threshold in the study by Mack et al. [70]. However, only 11 subjects were below the age of 18 , including five below the age of 16 , and profound hormonal changes, which might influence the development of the microbiome [73], emerge between the age of 12 and 15 years. Thus, it might be important to build on the results by Mack et al. [70] and also study younger adolescents (Fig. 1).

\section{Gut permeability, inflammation and anorexia nervosa}

A relationship between immune-mediated disorders and the gut microbiome is the subject of recent discussion. The microbiome stimulates the release of cytokines and other inflammatory mediators [11]. A "leaky gut", which can be provoked by starvation and increases in mucin-degrading bacteria, is characterized by antigens traversing the intestinal wall, infiltrating systemic circulation, and thus contributing to a chronic low-grade inflammation presumed to be present in AN. Interestingly, autoantibodies against neuropeptides associated with appetite and stress regulation have been identified in AN [74, 75]. Moreover, a Finnish study conducted between 1995 and 2010 with a large eating-disorder cohort $(n=2342)$ demonstrated an elevated risk for autoimmune diseases in these patients [50]. According to their dataset assessing 30 autoimmune diseases, the most frequent were of endocrinological and gastroenterological origin. The lifetime risk (OR) of patients with an eating disorder (all diagnoses) to also suffer from an endocrinological disease, mostly diabetes mellitus type 1 , was more than twice as high (OR 2.4) as for HC, while the risk of suffering from a gastroenterological disease was nearly twice as high (OR 1.8). The highest rates were found for an association with Crohn's disease (OR 3.09). In other previous studies, pro-inflammatory cytokines were shown to be increased in acute AN, although they mostly returned to normal after nutritional rehabilitation [76] (meta-analysis by Solmi et al. [77]). However, they may play a pivotal role in primarily chronic courses. In a recent case report of a young woman with co-existing Crohn's disease and AN, the latter was much improved by the prescription of antiTNF-alpha treatment [78].

Similar to a putative role in irritable bowel syndrome, increased gut permeability might also be associated with gastrointestinal complaints frequently occurring in acute and chronic AN [79, 80]. However, in the study by Mack et al. [70], gastrointestinal symptoms improved, but did not completely alleviate with weight gain, although microbial richness increased.

\section{Implications for treatment}

As discussed above, there is substantial evidence for alterations of the gut microbiome in eating disorders, especially AN. These new insights might lend support for new therapeutic targets in this often chronic disorder, such as defining the right target weight, modifications of the refeeding process and the composition of the diet, non-bacterial dietary supplements and possibly the future use of pre- or probiotics (see below).

\section{Target weight}

Although restoration of a healthy body weight is an important goal in the treatment of AN, there is no empirically derived agreement on the definition of an appropriate target weight (for a review, see [81]). However, with regard to body mass index (BMI), the gut microbiome plays an important role. Million et al. [82] analysed the association between the gut microbiome and body weight and found that the proportion of certain bacteria was significantly correlated with BMI. They compared four bacterial species in the following four weight classes: obese, overweight, lean and anorectic individuals. The abundance of Lactobacillus species was positively correlated with BMI, while Bifidobacterium animalis, Methanobrevibacter smithii and Escherichia coli were negatively correlated with BMI. Thus, given the differences observed in previous studies of AN (i.e. an increase in mucin producers and decrease in SFA-producers, [70]), it might be possible that the composition of the gut microbiome could be used to help determine individualized target weights for patients (e.g. in the context of a restoration of bacterial community structure).

\section{Refeeding and composition of diet}

Refeeding practices in AN are mostly based on mainstream clinical or expert opinions-there is little empirical evidence for a healthy weight regimen in affected individuals. While European clinicians usually start patients on a low-calorie diet to avoid the so-called refeeding syndrome, American practitioners are less cautious and suggest a much higher calorie supply even at the beginning of refeeding (for a review, see [81]).

According to the findings of the Kaye group [83], patients with the restricting type of AN need a higher quantity of calories to gain the same amount of weight as patients with the bulimic type. While this difference is 
mostly ascribed to higher energy expenditure in patients with the restrictive type compared to those with the bulimic type, additional involvement of the gut microbiome is conceivable. Indeed, Mack et al. [70] confirmed significant differences in microbial community composition according to AN subtype. Binge/purge patients often have a higher premorbid body weight, which might be associated with a different composition of the gut microbiome. The latter is a key determinant of the transformation of bile acids to efficiently take up lipids, carbohydrates and fatty acids [84]. The microbiome of both obese mice and humans has an increased capacity to harvest energy from nourishment [57, 85-87]. Faecal samples transplanted to GF mice from obese humans led to increased body and fat mass accompanied with obesity-associated metabolic dysfunctions [58]. However, normalization of body weight and obesityrelated metabolic perturbations was achievable through cohousing with lean mice (and hence, exposure to their gut microbes through coprophagy) combined with a diet rich in vegetables and fruit but low in saturated fats. Thus, one might speculate that augmenting gut microbial composition in combination with certain diets may have potential to improve weight restoration in AN.

The composition of the human microbiome has been linked to long-term dietary patterns, such as diets in Western (animal protein, sugar and fat) and Non-Western populations (plant-derived carbohydrates) [80, 88]. However, the human gut microbiome seems to respond rapidly to rigorous short-term macronutrient changes, whereby individual diet-driven changes can occur within 3-4 days, but reverse in a similar time frame [89]. In an important study by David et al. [90], the authors compared the effects of a shift in macronutrient intake from usual eating habits to either an animal-based diet or a plant-derived diet. Changes in the composition of the gut microbiome occurred only one day after the new diet had reached the distal gut, but exclusively in the group on the animal-based diet, and the gut microbiome returned to its usual composition two days after this diet ended. Although there was no difference in alpha diversity between the two groups, beta diversity (difference in community structure between baseline and diet) significantly changed in the animal-based food group. Notably, in contrast to the participants on the vegetarian diet, those on the animal-based diet showed significant weight loss, although caloric intake was similar in both groups. One of the most abundant phyla in the microbiota of the animal-based diet group was a bacterium with a high bile resistance (Bilophila wadsworthia), which is in accordance with our knowledge that a diet rich in fat results in higher bile acid production. In mice, growth of $B$. wadsworthia is stimulated by ingesting saturated fats from milk; it is important that the expansion rate of $B$. wadsworthia is also associated with the development of inflammatory bowel disease in these mouse models [90, 91]. Thus, we may hypothesize that certain diets-also in humans-will contribute to the pathogenesis of bowel inflammation.

Why are these findings important for the treatment of AN? Before admission, anorexia nervosa patients often maintain a vegetarian diet low in fat and high in fibre [92]. After hospitalization, diet is often quickly changed to a high-calorie diet rich in carbohydrates and fat for nutritional rehabilitation. Moreover, in cases of very severe AN, patients are sometimes tube-fed or are given oral liquid supplements. Most oral liquid supplements, especially those suitable for tube feeding, are based on cow's milk, e.g. an animal-based food product. Thus, within a very short time, we may strongly affect the gut microbiome of our patients without being aware of the consequences, such as the possible growth of inflammation-inducing bacteria. Although two studies investigated the impact of weight gain on the gut microbiota (see above, [69, 70]), none so far has explored the effect of the diet itself.

\section{Pre- and probiotics}

Gibson and Roberfroid [93] defined a prebiotic as a "nondigestible food ingredient that beneficially affects the host by selectively stimulating the growth and/or activity of one or a limited number of bacteria in the gut". Currently, the selectivity criterion is left out, and a prebiotic is defined as a "substance that induces the growth of microorganisms that contribute to the well-being of their host" (Wikipedia, assessed 7/7/2016 [94]; for a review, see [95]). The most well-known prebiotics to impact the gut-brain axis are fructans (such as inulins and oligofructose) and glucans (such as galacto-oligosaccharides) [11]. The latter have been demonstrated to improve intestinal barrier function in rodents [96]. More recently, substances such as pectins and milk oligosaccharides are also included among prebiotics. It is hypothesized that metabolic function of the organism can be improved and low-grade inflammation reduced by altering the gut microbiome with the help of prebiotics [87].

Other studies suggest that prebiotics may be effective in the treatment of depressive or anxious states and cognitive function. Glucans and polydextrose were shown to reduce anxiety-related behaviour in rats [97] and to attenuate stress response in healthy adult probands [98].

A probiotic is defined as living component of the microbiota administered to humans or animals that are associated with health benefits for the host. In several studies, a reduction of intestinal permeability was reported, e.g. in pre-term infants, in children with atopic dermatitis and irritable bowel syndrome after application of probiotics (for a review, see [12]). Möhle et al. [46] showed that 
after severely reducing the gut microbiome with several antibiotics, neuropsychological deficits, such as learning impairments and reduced neurogenesis in the hippocampus, could be induced in mice. These impairments could be reversed by orally administering an over-the-counter mix of probiotic bacteria. Although an effect of probiotics on depressive- and anxiety-like behaviour in mice has been observed [99], reports involving larger human samples are still lacking. Mazurak et al. [100] analysed the evidence of probiotic use in irritable bowel syndrome and criticised that studies suffer from heterogeneity in sample sizes, duration of treatment and concentration of probiotics. In a systematic review by Kristensen [101] including seven studies between 2013 and 2015, no significant effect of probiotics on faecal microbiota composition in healthy adults was found when compared to placebo. Moreover, there are no studies on the benefit of pre- or probiotics in AN.

It is beyond the scope of this article to discuss the benefit of pre- or probiotics in more detail. Sheridan and coauthors [102] assert that the type of malnutrition should be strictly defined and the target of the intervention should be specified before administering pre- or probiotics to undernourished patients. In addition, it should be kept in mind that there are differences in the type of malnutrition caused by famine, old age or somatic disorders in comparison to AN patients, who commonly ingest "healthy low caloric food" such as a vegetarian diet, which will most likely also result in different effects on the microbiome.

\section{Conclusions}

There is growing evidence that the gut microbiome plays a notable role in the emergence and development of somatic and psychological symptoms in AN. The consideration of microbe-gut-brain interactions as risk factors for the onset and perpetuation of AN and as a target for therapeutic interventions, will likely prove to be a significant shift in our scientific concept of the aetiology and treatment of eating disorders. Until now, there have been very few studies on the role of the intestinal microbiome in $\mathrm{AN}$ and other eating disorders. Accordingly, we are not aware of the consequences of a diet rapidly changing in caloric content and composition. Future research should include continuous monitoring of the microbiome during nutritional rehabilitation, weight gain and hormonal restoration far beyond previous assessments at only the beginning and end of treatment. Our knowledge on the effect of rapid weight gain, changes in macronutrients and target weight level on the microbiome and consequent inflammation mechanisms is scant. Moreover, our insight into gut-brain interaction, especially in adolescent AN, is very limited. However, the exploration of gut-brain communication and its alteration, especially in the early stages of the disease in adolescence, may be particularly important for the prognosis of this often long lasting and disabling disorder.

\section{Compliance with ethical standards}

Conflict of interest On behalf of all authors, the corresponding author states that there is no conflict of interest.

Open Access This article is distributed under the terms of the Creative Commons Attribution 4.0 International License (http://creativecommons.org/licenses/by/4.0/), which permits unrestricted use, distribution, and reproduction in any medium, provided you give appropriate credit to the original author(s) and the source, provide a link to the Creative Commons license, and indicate if changes were made.

\section{References}

1. Simopoulos AP (2001) The Hippocratic concept of positive health in the fifth century BC and in the new millennium. World Rev Nutr Diet 89:1-4

2. American Psychiatric Association (2013) Diagnostic and Statistical Manual of Mental Disorders (DSM-5). American Psychiatric Press

3. Minuchin S, Baker L, Rosman BL, Liebman R, Milman L, Todd TC (1975) A conceptual model of psychosomatic illness in children. Family organization and family therapy. Arch Gen Psychiatry 32:1031-1038

4. Herpertz-Dahlmann B, Seitz J, Konrad K (2011) Aetiology of anorexia nervosa: from a "psychosomatic family model" to a neuropsychiatric disorder? Eur Arch Psychiatry Clin Neurosci 261(Suppl 2):S177-S181. doi:10.1007/ s00406-011-0246-y

5. Kaplan AS, Walsh BT, Olmsted M, Attia E, Carter JC, Devlin MJ, Pike KM, Woodside B, Rockert W, Roberto CA, Parides M (2009) The slippery slope: prediction of successful weight maintenance in anorexia nervosa. Psychol Med 39:1037-1045. doi:10.1017/s003329170800442x

6. Misra M, Golden NH, Katzman DK (2016) State of the art systematic review of bone disease in anorexia nervosa. Int $\mathrm{J}$ Eat Disord 49:276-292. doi:10.1002/eat.22451

7. Misra M, Klibanski A (2016) Anorexia nervosa and its associated endocrinopathy in young people. Horm Res Paediatr 85:147-157. doi:10.1159/000443735

8. Pollice C, Kaye WH, Greeno CG, Weltzin TE (1997) Relationship of depression, anxiety, and obsessionality to state of illness in anorexia nervosa. Int J Eat Disord 21:367-376

9. Meehan KG, Loeb KL, Roberto CA, Attia E (2006) Mood change during weight restoration in patients with anorexia nervosa. Int J Eat Disord 39:587-589. doi:10.1002/eat.20337

10. Chen J, Papies EK, Barsalou LW (2016) A core eating network and its modulations underlie diverse eating phenomena. Brain Cogn. doi:10.1016/j.bandc.2016.04.004

11. Sherwin E, Rea K, Dinan TG, Cryan JF (2016) A gut (microbiome) feeling about the brain. Curr Opin Gastroenterol 32:96102. doi: $10.1097 / \operatorname{mog} .0000000000000244$

12. Kelly JR, Kennedy PJ, Cryan JF, Dinan TG, Clarke G, Hyland NP (2015) Breaking down the barriers: the gut microbiome, intestinal permeability and stress-related psychiatric disorders. Front Cell Neurosci 9:392. doi:10.3389/fncel.2015.00392 
13. Backhed F, Ley RE, Sonnenburg JL, Peterson DA, Gordon JI (2005) Host-bacterial mutualism in the human intestine. Science 307:1915-1920. doi:10.1126/science.1104816

14. Derrien M, van Hylckama Vlieg JE (2015) Fate, activity, and impact of ingested bacteria within the human gut microbiota. Trends Microbiol 23:354-366. doi:10.1016/j.tim.2015.03.002

15. Petra AI, Panagiotidou S, Hatziagelaki E, Stewart JM, Conti P, Theoharides TC (2015) Gut-microbiota-brain axis and its effect on neuropsychiatric disorders with suspected immune dysregulation. Clin Ther 37:984-995. doi:10.1016/j. clinthera.2015.04.002

16. Carr J, Kleiman SC, Bulik CM, Bulik-Sullivan EC, Carroll IM (2016) Can attention to the intestinal microbiota improve understanding and treatment of anorexia nervosa? Expert Rev Gastroenterol Hepatol 10:565-569. doi:10.1586/17474124.2016.1166 953

17. Steinhausen HC (2002) The outcome of anorexia nervosa in the 20th century. Am J Psychiatry 159:1284-1293

18. Arcelus J, Mitchell AJ, Wales J, Nielsen S (2011) Mortality rates in patients with anorexia nervosa and other eating disorders. A meta-analysis of 36 studies. Arch Gen Psychiatry 68:724-731. doi:10.1001/archgenpsychiatry.2011.74

19. Herpertz-Dahlmann B, van Elburg A, Castro-Fornieles J, Schmidt U (2015) ESCAP Expert Paper: new developments in the diagnosis and treatment of adolescent anorexia nervosa-a European perspective. Eur Child Adolesc Psychiatry 24:11531167. doi:10.1007/s00787-015-0748-7

20. Schmidt U, Campbell IC (2013) Treatment of eating disorders can not remain 'brainless': the case for brain-directed treatments. Eur Eat Disord Rev 21:425-427. doi:10.1002/erv.2257

21. Staubach F, Kunzel S, Baines AC, Yee A, McGee BM, Backhed F, Baines JF, Johnsen JM (2012) Expression of the bloodgroup-related glycosyltransferase B4galnt2 influences the intestinal microbiota in mice. ISME J 6:1345-1355. doi:10.1038/ ismej.2011.204

22. Kelly JR, Clarke G, Cryan JF, Dinan TG (2016) Brain-gutmicrobiota axis: challenges for translation in psychiatry. Ann Epidemiol 26:366-372. doi:10.1016/j.annepidem.2016.02.008

23. Vanuytsel $\mathrm{T}$, van Wanrooy $\mathrm{S}$, Vanheel $\mathrm{H}$, Vanormelingen $\mathrm{C}$, Verschueren S, Houben E, Salim Rasoel S, Tomicronth J, Holvoet L, Farre R, Van Oudenhove L, Boeckxstaens G, Verbeke K, Tack J (2014) Psychological stress and corticotropin-releasing hormone increase intestinal permeability in humans by a mast cell-dependent mechanism. Gut 63:1293-1299. doi:10.1136/ gutjnl-2013-305690

24. Monteleone AM, Monteleone P, Serino I, Amodio R, Monaco F, Maj M (2016) Underweight subjects with anorexia nervosa have an enhanced salivary cortisol response not seen in weight restored subjects with anorexia nervosa. Psychoneuroendocrinology 70:118-121. doi:10.1016/j.psyneuen.2016.05.004

25. Schorr M, Lawson EA, Dichtel LE, Klibanski A, Miller KK (2015) Cortisol measures across the weight spectrum. J Clin Endocrinol Metab 100:3313-3321. doi:10.1210/jc.2015-2078

26. Jesus P, Ouelaa W, Francois M, Riachy L, Guerin C, Aziz M, Do Rego JC, Dechelotte P, Fetissov SO, Coeffier M (2014) Alteration of intestinal barrier function during activity-based anorexia in mice. Clin Nutr 33:1046-1053. doi:10.1016/j.clnu.2013.11.006

27. Yano JM, Yu K, Donaldson GP, Shastri GG, Ann P, Ma L, Nagler CR, Ismagilov RF, Mazmanian SK, Hsiao EY (2015) Indigenous bacteria from the gut microbiota regulate host serotonin biosynthesis. Cell 161:264-276. doi:10.1016/j.cell.2015.02.047

28. Wikoff WR, Anfora AT, Liu J, Schultz PG, Lesley SA, Peters EC, Siuzdak G (2009) Metabolomics analysis reveals large effects of gut microflora on mammalian blood metabolites. Proc Natl Acad Sci 106:3698-3703. doi:10.1073/pnas.0812874106
29. Ridaura V, Belkaid Y (2015) Gut microbiota: the link to your second brain. Cell 161:193-194. doi:10.1016/j.cell.2015.03.033

30. Jenkins TA, Nguyen JC, Polglaze KE, Bertrand PP (2016) Influence of tryptophan and serotonin on mood and cognition with a possible role of the gut-brain axis. Nutrients. doi:10.3390/ nu8010056

31. Neufeld KM, Kang N, Bienenstock J, Foster JA (2011) Reduced anxiety-like behavior and central neurochemical change in germ-free mice. Neurogastroenterol Motil 23(255-264):e119. doi:10.1111/j.1365-2982.2010.01620.x

32. Duman RS (2011) Neurochemical theories of depression: preclinical studies. In: Charney DS, Nestler EJ (eds) Neurobiology of mental illness. Oxford Press, pp 414-444

33. Clarke G, Grenham S, Scully P, Fitzgerald P, Moloney RD, Shanahan F, Dinan TG, Cryan JF (2013) The microbiome-gutbrain axis during early life regulates the hippocampal serotonergic system in a sex-dependent manner. Mol Psychiatry 18:666673. doi:10.1038/mp.2012.77

34. Jiang H, Ling Z, Zhang Y, Mao H, Ma Z, Yin Y, Wang W, Tang W, Tan Z, Shi J, Li L, Ruan B (2015) Altered fecal microbiota composition in patients with major depressive disorder. Brain Behav Immun 48:186-194. doi:10.1016/j.bbi.2015.03.016

35. Lurie I, Yang YX, Haynes K, Mamtani R, Boursi B (2015) Antibiotic exposure and the risk for depression, anxiety, or psychosis: a nested case-control study. J Clin Psychiatry 76:15221528. doi:10.4088/JCP.15m09961

36. Kaye WH, Fudge JL, Paulus M (2009) New insights into symptoms and neurocircuit function of anorexia nervosa. Nat Rev Neurosci 10:573-584. doi:10.1038/nrn2682

37. Kaye WH, Wierenga CE, Bailer UF, Simmons AN, BischoffGrethe A (2013) Nothing tastes as good as skinny feels: the neurobiology of anorexia nervosa. Trends Neurosci 36:110 120. doi:10.1016/j.tins.2013.01.003

38. Herpertz-Dahlmann B, Wewetzer C (2014) Eating disorders. In: Gerlach M, Warnke A, Greenhill L (eds) Psychiatric drugs in children and adolescents. Springer, Heidelberg, pp 429-437

39. Harrisberger F, Smieskova R, Schmidt A, Lenz C, Walter A, Wittfeld K, Grabe HJ, Lang UE, Fusar-Poli P, Borgwardt S (2015) BDNF Val66Met polymorphism and hippocampal volume in neuropsychiatric disorders: a systematic review and meta-analysis. Neurosci Biobehav Rev 55:107-118. doi:10.1016/j.neubiorev.2015.04.017

40. Desbonnet L, Clarke G, Traplin A, O’Sullivan O, Crispie F, Moloney RD, Cotter PD, Dinan TG, Cryan JF (2015) Gut microbiota depletion from early adolescence in mice: implications for brain and behaviour. Brain Behav Immun 48:165-173. doi:10.1016/j.bbi.2015.04.004

41. Savignac HM, Corona G, Mills H, Chen L, Spencer JP, Tzortzis G, Burnet PW (2013) Prebiotic feeding elevates central brain derived neurotrophic factor, $N$-methyl-D-aspartate receptor subunits and D-serine. Neurochem Int 63:756-764. doi:10.1016/j. neuint.2013.10.006

42. Liang S, Wang T, Hu X, Luo J, Li W, Wu X, Duan Y, Jin F (2015) Administration of Lactobacillus helveticus NS8 improves behavioral, cognitive, and biochemical aberrations caused by chronic restraint stress. Neuroscience 310:561-577. doi:10.1016/j.neuroscience.2015.09.033

43. Savignac HM, Tramullas M, Kiely B, Dinan TG, Cryan JF (2015) Bifidobacteria modulate cognitive processes in an anxious mouse strain. Behav Brain Res 287:59-72. doi:10.1016/j. bbr.2015.02.044

44. Monteleone P, Fabrazzo M, Martiadis V, Serritella C, Pannuto M, Maj M (2005) Circulating brain-derived neurotrophic factor is decreased in women with anorexia and bulimia nervosa but not in women with binge-eating disorder: relationships to 
co-morbid depression, psychopathology and hormonal variables. Psychol Med 35:897-905

45. Zwipp J, Hass J, Schober I, Geisler D, Ritschel F, Seidel M, Weiss J, Roessner V, Hellweg R, Ehrlich S (2014) Serum brain-derived neurotrophic factor and cognitive functioning in underweight, weight-recovered and partially weight-recovered females with anorexia nervosa. Prog Neuropsychopharmacol Biol Psychiatry 54:163-169. doi:10.1016/j.pnpbp.2014.05.006

46. Mohle L, Mattei D, Heimesaat MM, Bereswill S, Fischer A, Alutis M, French T, Hambardzumyan D, Matzinger P, Dunay IR, Wolf SA (2016) Ly6C(hi) monocytes provide a link between antibiotic-induced changes in gut microbiota and adult hippocampal neurogenesis. Cell Rep 15:1945-1956. doi:10.1016/j. celrep.2016.04.074

47. Martin-Subero M, Anderson G, Kanchanatawan B, Berk M, Maes M (2016) Comorbidity between depression and inflammatory bowel disease explained by immune-inflammatory, oxidative, and nitrosative stress; tryptophan catabolite; and gut-brain pathways. CNS Spectr 21:184-198. doi:10.1017/ s1092852915000449

48. Bailey MT, Dowd SE, Galley JD, Hufnagle AR, Allen RG, Lyte M (2011) Exposure to a social stressor alters the structure of the intestinal microbiota: implications for stressor-induced immunomodulation. Brain Behav Immun 25:397-407. doi:10.1016/j. bbi.2010.10.023

49. Maes M, Kubera M, Leunis JC, Berk M (2012) Increased IgA and IgM responses against gut commensals in chronic depression: further evidence for increased bacterial translocation or leaky gut. J Affect Disord 141:55-62. doi:10.1016/j. jad.2012.02.023

50. Raevuori A, Haukka J, Vaarala O, Suvisaari JM, Gissler M, Grainger M, Linna MS, Suokas JT (2014) The increased risk for autoimmune diseases in patients with eating disorders. PLoS One 9:e104845. doi:10.1371/journal.pone.0104845

51. Frost GS, Walton GE, Swann JR, Psichas A, Costabile A, Johnson LP, Sponheimer M, Gibson GR, Barraclough TG (2014) Impacts of plant-based foods in ancestral hominin diets on the metabolism and function of gut microbiota in vitro. MBio 5:e00853-e00814. doi:10.1128/mBio.00853-14

52. den Besten G, van Eunen K, Groen AK, Venema K, Reijngoud DJ, Bakker BM (2013) The role of short-chain fatty acids in the interplay between diet, gut microbiota, and host energy metabolism. J Lipid Res 54:2325-2340. doi:10.1194/jlr.R036012

53. Stilling RM, Bordenstein SR, Dinan TG, Cryan JF (2014) Friends with social benefits: host-microbe interactions as a driver of brain evolution and development? Front Cell Infect Microbiol 4:147. doi:10.3389/fcimb.2014.00147

54. Stilling RM, Dinan TG, Cryan JF (2014) Microbial genes, brain \& behaviour - epigenetic regulation of the gut-brain axis. Genes Brain Behav 13:69-86. doi:10.1111/gbb.12109

55. De Vadder F, Kovatcheva-Datchary P, Goncalves D, Vinera J, Zitoun C, Duchampt A, Backhed F, Mithieux G (2014) Microbiota-generated metabolites promote metabolic benefits via gut-brain neural circuits. Cell 156:84-96. doi:10.1016/j. cell.2013.12.016

56. Byrne CS, Chambers ES, Morrison DJ, Frost G (2015) The role of short chain fatty acids in appetite regulation and energy homeostasis. Int J Obes (Lond) 39:1331-1338. doi:10.1038/ ijo.2015.84

57. Turnbaugh PJ, Ley RE, Mahowald MA, Magrini V, Mardis ER, Gordon JI (2006) An obesity-associated gut microbiome with increased capacity for energy harvest. Nature 444:1027-1031. doi:10.1038/nature05414

58. Ridaura VK, Faith JJ, Rey FE, Cheng J, Duncan AE, Kau AL, Griffin NW, Lombard V, Henrissat B, Bain JR, Muehlbauer MJ, Ilkayeva O, Semenkovich CF, Funai K, Hayashi DK, Lyle
BJ, Martini MC, Ursell LK, Clemente JC, Van Treuren W, Walters WA, Knight R, Newgard CB, Heath AC, Gordon JI (2013) Gut microbiota from twins discordant for obesity modulate metabolism in mice. Science 341:1241214. doi:10.1126/ science. 1241214

59. Smith MI, Yatsunenko T, Manary MJ, Trehan I, Mkakosya R, Cheng J, Kau AL, Rich SS, Concannon P, Mychaleckyj JC, Liu J, Houpt E, Li JV, Holmes E, Nicholson J, Knights D, Ursell LK, Knight R, Gordon JI (2013) Gut microbiomes of Malawian twin pairs discordant for kwashiorkor. Science 339:548-554. doi:10.1126/science. 1229000

60. Adan RA, Hillebrand JJ, De Rijke C, Nijenhuis W, Vink T, Garner KM, Kas MJ (2003) Melanocortin system and eating disorders. Ann N Y Acad Sci 994:267-274

61. Monteleone P, Carratu R, Carteni M, Generoso M, Lamberti M, Magistris LD, Brambilla F, Colurcio B, Secondulfo M, Maj M (2004) Intestinal permeability is decreased in anorexia nervosa. Mol Psychiatry 9:76-80. doi:10.1038/sj.mp.4001374

62. Uil JJ, van Elburg RM, van Overbeek FM, Mulder CJ, VanBergeHenegouwen GP, Heymans HS (1997) Clinical implications of the sugar absorption test: intestinal permeability test to assess mucosal barrier function. Scand J Gastroenterol Suppl 223:70-78

63. Pals KL, Chang RT, Ryan AJ, Gisolfi CV (1997) Effect of running intensity on intestinal permeability. J Appl Physiol (1985) 82:571-576

64. Elia M, Behrens R, Northrop C, Wraight P, Neale G (1987) Evaluation of mannitol, lactulose and 51Cr-labelled ethylenediaminetetra-acetate as markers of intestinal permeability in man. Clin Sci (Lond) 73:197-204

65. Welsh FK, Farmery SM, MacLennan K, Sheridan MB, Barclay GR, Guillou PJ, Reynolds JV (1998) Gut barrier function in malnourished patients. Gut 42:396-401

66. Pfleiderer A, Lagier JC, Armougom F, Robert C, Vialettes B, Raoult D (2013) Culturomics identified 11 new bacterial species from a single anorexia nervosa stool sample. Eur J Clin Microbiol Infect Dis 32:1471-1481. doi:10.1007/s10096-013-1900-2

67. Morita H, Obata K, Abe C, Shiba D, Shirakawa M, Kudo T, Takahashi S (2015) Feasibility of a short-arm centrifuge for mouse hypergravity experiments. PLoS One 10:e0133981. doi:10.1371/journal.pone.0133981

68. Armougom F, Henry M, Vialettes B, Raccah D, Raoult D (2009) Monitoring bacterial community of human gut microbiota reveals an increase in Lactobacillus in obese patients and methanogens in anorexic patients. PLoS One 4:e7125. doi:10.1371/journal.pone.0007125

69. Kleiman SC, Watson HJ, Bulik-Sullivan EC, Huh EY, Tarantino LM, Bulik CM, Carroll IM (2015) The intestinal microbiota in acute anorexia nervosa and during renourishment: relationship to depression, anxiety, and eating disorder psychopathology. Psychosom Med 77:969-981. doi:10.1097/ psy.0000000000000247

70. Mack I, Cuntz U, Gramer C, Niedermaier S, Pohl C, Schwiertz A, Zimmermann K, Zipfel S, Enck P, Penders J (2016) Weight gain in anorexia nervosa does not ameliorate the faecal microbiota, branched chain fatty acid profiles, and gastrointestinal complaints. Sci Rep 6:26752. doi:10.1038/srep26752

71. Holzer P, Farzi A (2014) Neuropeptides and the microbiota-gut-brain axis. Adv Exp Med Biol 817:195-219. doi:10.1007/978-1-4939-0897-4_9

72. Smink FR, van Hoeken D, Hoek HW (2013) Epidemiology, course, and outcome of eating disorders. Curr Opin Psychiatry 26:543-548. doi:10.1097/YCO.0b013e328365a24f

73. Neuman H, Debelius JW, Knight R, Koren O (2015) Microbial endocrinology: the interplay between the microbiota and the endocrine system. FEMS Microbiol Rev 39:509-521. doi:10.1093/femsre/fuu010 
74. Terashi M, Asakawa A, Harada T, Ushikai M, Coquerel Q, Sinno MH, Dechelotte P, Inui A, Fetissov SO (2011) Ghrelin reactive autoantibodies in restrictive anorexia nervosa. Nutrition 27:407-413. doi:10.1016/j.nut.2011.01.002

75. Fetissov SO, Crook MA (2011) What increased consumption of licorice may reveal in anorexia nervosa. Nutrition 27:853-854. doi:10.1016/j.nut.2011.05.004

76. Corcos M, Guilbaud O, Paterniti S, Moussa M, Chambry J, Chaouat G, Consoli SM, Jeammet P (2003) Involvement of cytokines in eating disorders: a critical review of the human literature. Psychoneuroendocrinology 28:229-249

77. Solmi M, Veronese N, Favaro A, Santonastaso P, Manzato E, Sergi G, Correll CU (2015) Inflammatory cytokines and anorexia nervosa: a meta-analysis of cross-sectional and longitudinal studies. Psychoneuroendocrinology 51:237-252. doi:10.1016/j.psyneuen.2014.09.031

78. Solmi M, Santonastaso P, Caccaro R, Favaro A (2013) A case of anorexia nervosa with comorbid Crohn's disease: beneficial effects of anti-TNF-alpha therapy? Int J Eat Disord 46:639641. doi:10.1002/eat.22153

79. Lee KN, Lee OY (2014) Intestinal microbiota in pathophysiology and management of irritable bowel syndrome. World J Gastroenterol 20:8886-8897. doi:10.3748/wjg.v20.i27.8886

80. Rajilic-Stojanovic M, Jonkers DM, Salonen A, Hanevik K, Raes J, Jalanka J, de Vos WM, Manichanh C, Golic N, Enck P, Philippou E, Iraqi FA, Clarke G, Spiller RC, Penders J (2015) Intestinal microbiota and diet in IBS: causes, consequences, or epiphenomena? Am J Gastroenterol 110:278-287. doi:10.1038/ ajg. 2014.427

81. Herpertz-Dahlmann B (2015) Adolescent eating disorders: update on definitions, symptomatology, epidemiology, and comorbidity. Child Adolesc Psychiatr Clin N Am 24:177-196. doi:10.1016/j.chc.2014.08.003

82. Million M, Angelakis E, Maraninchi M, Henry M, Giorgi R, Valero R, Vialettes B, Raoult D (2013) Correlation between body mass index and gut concentrations of Lactobacillus reuteri, Bifidobacterium animalis, Methanobrevibacter smithii and Escherichia coli. Int J Obes (Lond) 37:1460-1466. doi:10.1038/ ijo. 2013.20

83. Marzola E, Panepinto C, Delsedime N, Amianto F, Fassino S, Abbate-Daga G (2016) A factor analysis of the meanings of anorexia nervosa: intrapsychic, relational, and avoidant dimensions and their clinical correlates. BMC Psychiatry 16:190. doi:10.1186/s12888-016-0894-6

84. Kleiman SC, Carroll IM, Tarantino LM, Bulik CM (2015) Gut feelings: a role for the intestinal microbiota in anorexia nervosa? Int J Eat Disord 48:449-451. doi:10.1002/eat.22394

85. Goffredo M, Mass K, Parks EJ, Wagner DA, McClure EA, Graf J, Savoye M, Pierpont B, Cline G, Santoro N (2016) Role of gut microbiota and short chain fatty acids in modulating energy harvest and fat partitioning in youth. J Clin Endocrinol Metab 101:4367-4376. doi:10.1210/jc.2016-1797

86. Jumpertz R, Le DS, Turnbaugh PJ, Trinidad C, Bogardus C, Gordon JI, Krakoff J (2011) Energy-balance studies reveal associations between gut microbes, caloric load, and nutrient absorption in humans. Am J Clin Nutr 94:58-65. doi:10.3945/ ajen.110.010132

87. Martinez KB, Pierre JF, Chang EB (2016) The gut microbiota: the gateway to improved metabolism. Gastroenterol Clin N Am 45:601-614. doi:10.1016/j.gtc.2016.07.001

88. Salonen A, de Vos WM (2014) Impact of diet on human intestinal microbiota and health. Annu Rev Food Sci Technol 5:239262. doi:10.1146/annurev-food-030212-182554
89. Walker AW, Ince J, Duncan SH, Webster LM, Holtrop G, Ze X, Brown D, Stares MD, Scott P, Bergerat A, Louis P, McIntosh F, Johnstone AM, Lobley GE, Parkhill J, Flint HJ (2011) Dominant and diet-responsive groups of bacteria within the human colonic microbiota. ISME J 5:220-230. doi:10.1038/ ismej.2010.118

90. David LA, Maurice CF, Carmody RN, Gootenberg DB, Button JE, Wolfe BE, Ling AV, Devlin AS, Varma Y, Fischbach MA, Biddinger SB, Dutton RJ, Turnbaugh PJ (2014) Diet rapidly and reproducibly alters the human gut microbiome. Nature 505:559-563. doi:10.1038/nature12820

91. Devkota S, Wang Y, Musch MW, Leone V, Fehlner-Peach H, Nadimpalli A, Antonopoulos DA, Jabri B, Chang EB (2012) Dietary-fat-induced taurocholic acid promotes pathobiont expansion and colitis in I110-/- mice. Nature 487:104-108. doi:10.1038/nature11225

92. Misra M, Tsai P, Anderson EJ, Hubbard JL, Gallagher K, Soyka LA, Miller KK, Herzog DB, Klibanski A (2006) Nutrient intake in community-dwelling adolescent girls with anorexia nervosa and in healthy adolescents. Am J Clin Nutr 84:698-706

93. Gibson GR, Roberfroid MB (1995) Dietary modulation of the human colonic microbiota: introducing the concept of prebiotics. J Nutr 125:1401-1412

94. https://en.wikipedia.org/wiki/Wikipedia:WikiProject_Wikipedia/ Assessment

95. Hutkins RW, Krumbeck JA, Bindels LB, Cani PD, Fahey G Jr, Goh YJ, Hamaker B, Martens EC, Mills DA, Rastal RA, Vaughan E, Sanders ME (2016) Prebiotics: why definitions matter. Curr Opin Biotechnol 37:1-7. doi:10.1016/j. copbio.2015.09.001

96. Zhong Y, Cai D, Cai W, Geng S, Chen L, Han T (2009) Protective effect of galactooligosaccharide-supplemented enteral nutrition on intestinal barrier function in rats with severe acute pancreatitis. Clin Nutr 28:575-580. doi:10.1016/j. clnu.2009.04.026

97. Mika A, Greenwood B N, Chichlowski M, Borchert D, Hulen K A, Berg B et al (2014) Dietary prebiotics increase Bifidobacterium spp. and Lactobacillus spp. in the gut and promote stress resistance. Brain Behav Immun 40(Supplement). doi:10.1016/j. bbi.2014.06.175

98. Schmidt K, Cowen PJ, Harmer CJ, Tzortzis G, Errington S, Burnet PW (2015) Prebiotic intake reduces the waking cortisol response and alters emotional bias in healthy volunteers. Psychopharmacology 232:1793-1801. doi:10.1007/ s00213-014-3810-0

99. Savignac HM, Kiely B, Dinan TG, Cryan JF (2014) Bifidobacteria exert strain-specific effects on stress-related behavior and physiology in BALB/c mice. Neurogastroenterol Motil 26:1615-1627. doi:10.1111/nmo.12427

100. Mazurak N, Broelz E, Storr M, Enck P (2015) Probiotic therapy of the irritable bowel syndrome: why is the evidence still poor and what can be done about It? J Neurogastroenterol Motil 21:471-485. doi:10.5056/jnm15071

101. Kristensen NB, Bryrup T, Allin KH, Nielsen T, Hansen TH, Pedersen O (2016) Alterations in fecal microbiota composition by probiotic supplementation in healthy adults: a systematic review of randomized controlled trials. Genome Med 8:52. doi:10.1186/s13073-016-0300-5

102. Sheridan PO, Bindels LB, Saulnier DM, Reid G, Nova E, Holmgren K, O'Toole PW, Bunn J, Delzenne N, Scott KP (2014) Can prebiotics and probiotics improve therapeutic outcomes for undernourished individuals? Gut Microbes 5:74-82. doi:10.4161/gmic. 27252 